\title{
Total ureterectomy and ileal ureteric replacement for TCC ureter in a solitary kidney
}

\author{
John S. Banerji, MD; ${ }^{*+}$ Arun J.P. George, MS, MCh ${ }^{*}$ \\ *Christian Medical College, Vellore, India; tVirginia Mason Medical Center, Seattle, Washington
}

Cite as: Can Urol Assoc J 2014;8(11-12):e938-40. http://dx.doi.org/10.5489/cuaj.2255 Published online December 15, 2014.

\section{Abstract}

Traditional treatment of upper tract transitional cell carcinoma (TCC) is nephroureterectomy with a bladder cuff. This is in keeping with the nature of the disease, in that TCC is a panurothelial disease. However, there are a few rare occasions when this would mean making a subject anephric, as in a TCC in a solitary kidney or bilateral synchronous/metachronous disease. We present a case of a patient with a dysplastic, poorly functioning left kidney and with a TCC of the ureter on the right side.

\section{Introduction}

Management of upper tract transitional cell carcinoma (TCC) of a high grade and stage in a single kidney can be challenging. Radical nephroureterectomy would place the patient in chronic kidney disease stage $\mathrm{V}$, requiring renal replacement therapy. Oncologically however, it is evident from studies that segmental or total ureterectomy is not inferior to radical nephroureterectomy. ${ }^{1}$

\section{Case report}

A 50-year-old, non-diabetic male, with a long history of smoking, presented with recurrent episodes of gross painless hematuria. This was associated with occasional urgency and burning micturition.

His urine microscopy showed numerous red blood cells and leucocytes. His urine culture was sterile. His initial creatinine was $1.6 \mathrm{mg} / \mathrm{dL}$. Urine cytology was positive. An ultrasound revealed right hydroureteronephrosis, and a small, dysplastic left kidney.

Ureteroscopy revealed an ulcerated area of about $2 \mathrm{~cm}$ in length, at the mid-ureteric region, with apparently normal mucosa proximally and distally. The ureter was involved almost circumferentially. The proximal ureter and entire pelvicalyceal system were inspected with a flexible ureteroscope to ensure there were no synchronous tumours or suspicious areas of carcinoma in situ. An ureteroscopic biopsy was taken which confirmed high-grade TCC.

Axial imaging in the form of a magnetic resonance imaging (MRI) with a MR urogram was done, which revealed a 2-cm hypointense segment on a T2 weighted image, at the mid-ureter (Fig. 1).

He underwent a total ureterectomy (Fig. 2) and an extended lymphadenectomy from the renal hilum to the inferior mesenteric artery, and the ipsilateral iliac and pelvic nodes. An isoperistaltic segment of ileum was then fashioned to construct an ileal ureter.

An intravenous urogram done at 3 weeks after the procedure showed good drainage and absence of any leak (Fig. 3). The final histopathology revealed a pT3, high-grade TCC. However, all nodes were negative for malignancy.

In view of the extensive local disease, he subsequently received 4 cycles of gemcitabine as adjuvant chemotherapy.

Postoperatively, his renal function initially deteriorated, with his creatinine climbing to $1.8 \mathrm{mg} / \mathrm{dL}$. However, at the 3-month follow-up, his creatinine stabilized to $1.4 \mathrm{mg} / \mathrm{dL}$. The marginal improvement in creatinine, when compared to the preoperative value $(1.6 \mathrm{mg} / \mathrm{dL})$ was probably due to the relief of the obstruction, which was being caused by the circumferential mid-ureteric tumour.

At the 15-month follow-up he was tumour-free and he underwent contrast enhanced computed tomography with delayed images and a flexible ureteroscopy. He will continue to require bladder and upper tract surveillance with cross-sectional imaging and flexible ureteroscopies.

\section{Discussion}

The gold standard treatment for upper tract TCC remains radical nephroureterectomy. However, in rare situations, there is an absolute indication to preserve a functioning renal unit. It is in these rare cases that segmental ureterectomies 


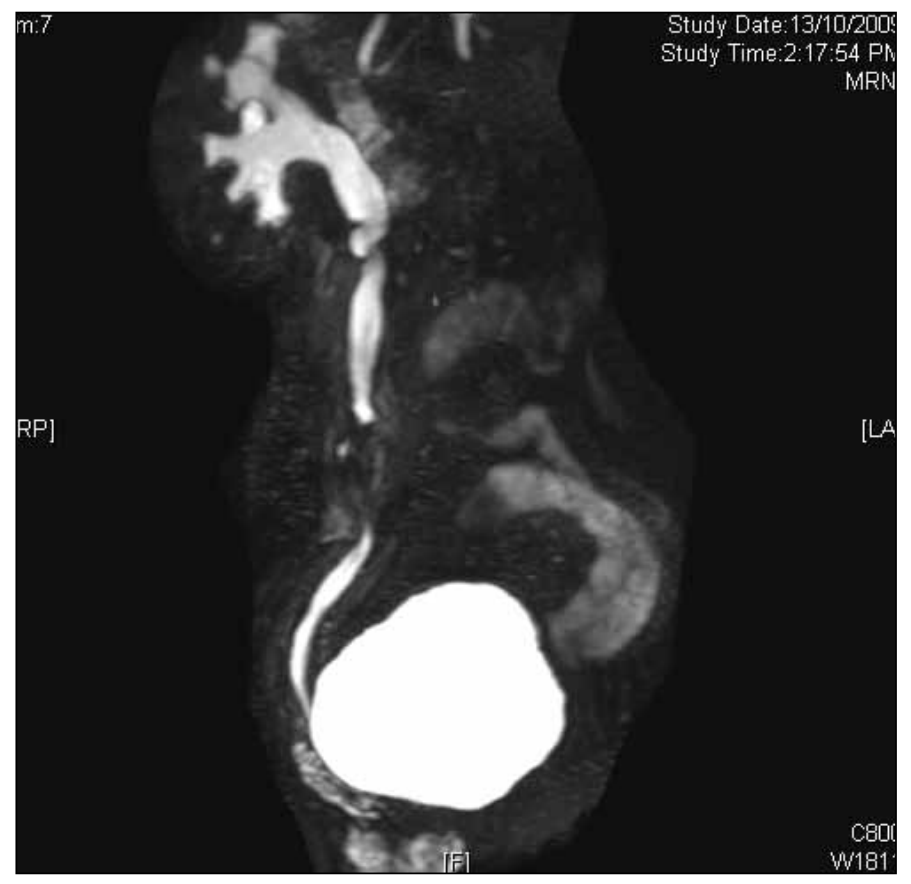

Fig. 1. T2 weighted magnetic resonance image showing hypointense lesion in the mid-ureteric segment, with obvious peri-ureteric thickening.

or total ureterectomy becomes mandatory.

In carefully selected patients with solitary ureteral lesions, outcomes following subtotal ureterectomy are similar to those of radical nephroureterectomy. Mazeman reported that the incidence of regional failure was similar for 246 patients treated by nephroureterectomy compared with 44 patients treated by distal ureterectomy. ${ }^{2}$ Recurrence rates in the ipsilateral ureter ranged from $10 \%$ to $25 \%$ and varied with pathologic stage and grade of the original tumour. Lowgrade, low-stage lesions had excellent 5-year outcomes, with ipsilateral ureteral recurrence rates of less than $5 \%$. In contrast, the prognosis for higher stage lesions was worse, with 5 -year survival rates of $65 \%$ for $\mathrm{T} 1$ lesions and $50 \%$ for T2 tumours.

Forwarding the case for partial ureterectomy, Pohar and Sheinfeld concluded that this was a viable proposition in patients with a solitary kidney, bilateral tumours, or renal insufficiency. ${ }^{3}$ This would obviate the need for dialysis, which not only affects quality of life but is associated with its own morbidity. The authors have gone a step further and stated that even those with grade 3 tumours of the upper and mid-ureter can be candidates for partial ureterectomy and reimplantation.

There have been reports of renal-sparing approaches for upper tract TCC in solitary kidneys. Milner and colleagues reported on the percutaneous management of low-grade tumours in a solitary kidney and concluded that in motivated patients, who would adhere to strict follow-up regimens, this was a viable option. ${ }^{4}$

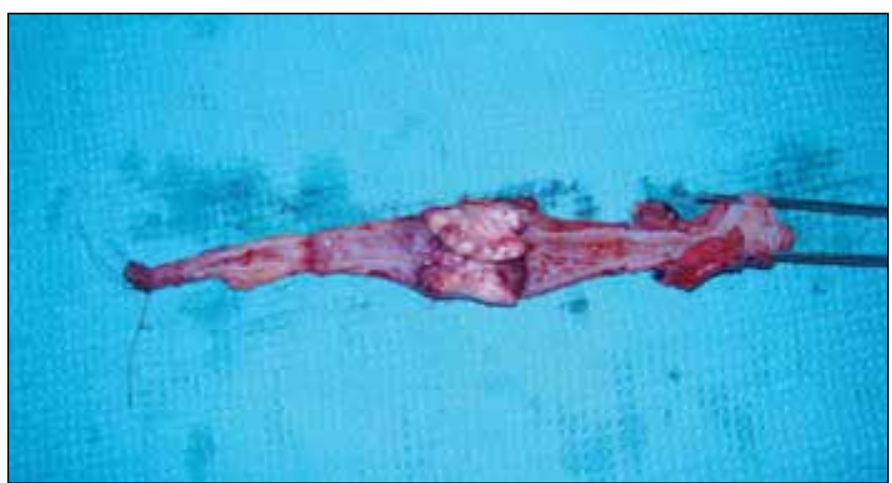

Fig. 2. Completely excised ureter, cut open longitudinally, revealing solid tumour.

Looking at this from a cost analysis perspective, Pak and colleagues concluded that renal-sparing upper urinary tract TCC management was effective at reducing healthcare expenses related to end-stage renal disease. ${ }^{4}$

Our patient was unique in that the tumour was unmanageable endoscopically, but the option of radical nephroureterectomy would render him anephric. Had the tumour been a smaller, non-circumferential lesion, involving the

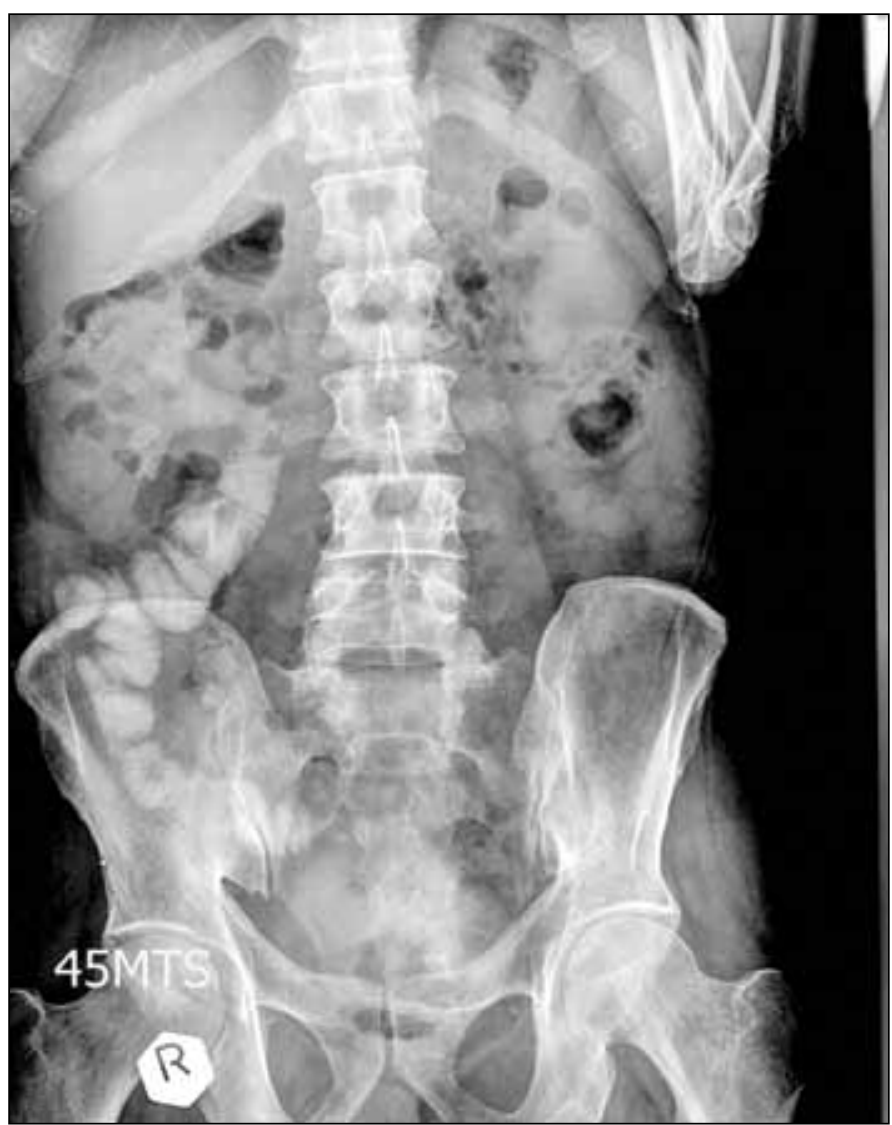

Fig. 3. Intravenous urogram (delayed - 45 minute film) showing ileal ureter and contrast in bladder, without evidence of any leak. 
Banerji et al.

mucosa and probably lamina propria alone, a conservative approach, like a segmental ureterectomy and a Boari flap, could have been performed. However, in our patient, the MRI clearly depicted that there was a good chance of periureteric infiltration by the tumour, which was subsequently confirmed on final histopathology.

Given that we were not performing a nephroureterectomy, the next best oncologically complete procedure was a total ureterectomy.

\section{Conclusion}

In a given situation upon careful selection, a total ureterectomy with ileal conduit, followed by adjuvant chemotherapy is a good option for clinically localized ureteric urothelial carcinoma.

Competing interests: Authors declare no competing financial or personal interests.
This paper has been peer-reviewed.

\section{References}

1. Simonato $A$, Varca $V$, Bianchi $G$, et al. Elective segmental ureterectomy for transitional cell carcinoma of the ureter: Long-term follow-up in a series of 73 patients. BJU Int 2012;1 10:E744-9. http://dx.doi. org/10.1111/j.1464

2. Mazeman E. Tumours of the upper urinary tract calyces, renal pelvis and ureter. Eur Urol 1976;2:120-6.

3. Pohar KS, Sheinfeld J. When is partial ureterectomy acceptable for transitional cell carcinoma of the ureter? J Endourol 2001;15:405-8. http://dx.doi.org/10.1089/089277901300189439

4. Milner JE, Voelzke BB, Flanigan RC, et al. Urothelial-cell carcinoma and solitary kidney: Outcomes with renal-sparing management. J Endourol 2006;20:800-7. http://dx.doi.org/10.1089/end.2006.20.800

5. Pak RW, Moskowitz EJ, Bagley DH. What is the cost of maintaining a kidney in upper-tract transitionalcell carcinoma? An objective analysis of cost and survival. J Endourol 2009;23:341-6. hittp://dx.doi. org/10.1089/end.2008.0251

Correspondence: Dr. John S. Banerii, Christian Medical College, Vellore, India; johnsbaneriן@cmcvellore.ac.in 